\title{
Uso del feedback correctivo indirecto metalingüístico y su efecto en la adquisición de aspectos gramaticales en estudiantes de pedagogía en inglés de una universidad chilena
}

Ortiz Navarrete, Mabel; Fuica, María Angélica; Sáez Sáez, Lorena

Uso del feedback correctivo indirecto metalingüístico y su efecto en la adquisición de aspectos

gramaticales en estudiantes de pedagogía en inglés de una universidad chilena

Revista Educación, vol. 43, núm. 2, 2019

Universidad de Costa Rica, Costa Rica

Disponible en: http://www.redalyc.org/articulo.oa?id=44058158024

DOI: https://doi.org/10.15517/revedu.v43i2.31933

Esta obra está bajo una Licencia Creative Commons Atribución-NoComercial-SinDerivar 3.0 Internacional. 


\section{Uso del feedback correctivo indirecto metalingüístico y su efecto en la adquisición de aspectos gramaticales en estudiantes de pedagogía en inglés de una universidad chilena}

Indirect Metalinguistic Corrective Feedback and its Effect on Grammar Acquisition in EFL Preservice Teachers at a Chilean University

Mabel Ortiz Navarrete

DOI: https://doi.org/10.15517/revedu.v43i2.31933

Universidad Católica de la Ssma Concepción, Concepción, Redalyc: http://www.redalyc.org/articulo.oa?id=44058158024 Chile

mortiz@ucsc.cl

(iD http://orcid.org/0000-0001-7481-1293

\section{Maria Angélica Fuica}

Universidad Católica de la Ssma Concepción, Concepción,

Chile

mfuica@ucsc.cl

(D) http://orcid.org/0000-0002-1584-0796

Lorena Sáez Sáez

Universidad Católica de la Ssma Concepción, Concepción,

Chile

1saez@ucsc.cl

(D) http://orcid.org/https://

orcid.org/0000-0002-6481-8683

Recepción: 24 Enero 2018

Aprobación: 22 Mayo 2019

\section{Resumen:}

El presente artículo tiene como objetivo dar a conocer los resultados de un estudio enfocado en el impacto del feedback correctivo indirecto metalingüístico en la adquisición de aspectos gramaticales en inglés. El diseño de la investigación correspondió a un preexperimento, cuya muestra fue constituida por 20 estudiantes de tercer año de un programa de formación de profesorado de inglés de una universidad chilena. El tratamiento consistió en la producción de cuatro ensayos argumentativos breves y la provisión de feedback correctivo indirecto sobre los errores gramaticales encontrados en cada uno de ellos. Después de la provisión de feedback, el estudiantado corregía sus errores y escribían un nuevo texto. Al comienzo y al final del tratamiento, se aplicó un test para observar si hubo una reducción en el número de errores gramaticales después de la intervención. Los resultados muestran que hubo una reducción estadísticamente significativa en el número de errores gramaticales después del tratamiento.

Palabras clave: Feedback correctivo indirecto, Adquisición, Texto argumentativo.

\section{Abstract:}

This article reveals finding from a study on the impact of indirect metalinguistic corrective feedback on English grammar acquisition. The study followed a pre-experimental design with a sample of 20 third-year students of a Program for Teaching English as a Foreign Language at a Chilean university. The sample group was asked to write four short argumentative essays for which they received indirect corrective feedback pertaining to grammatical errors observed and then proceeded to rewrite the essays based on the feedback provided. Students were also tested before and after completion of the study, revealing that the number of grammatical errors in their writing composition significantly declined in the latter situation.

KEYWORDS: Indirect Corrective Feedback, Acquisition, Argumentative Text. 


\section{INTRODUCCIÓN}

La producción escrita es una de las habilidades lingüísticas más difíciles de desarrollar (Kessler, 2009; Myles, 2002; Warschauer, 2010), puesto que "es una tarea compleja que debe coordinar varias actividades mentales" (Arias y García, 2006, p. 38) Dicha complejidad, propia de la escritura, al parecer se ve incrementada en los casos en que se estudia una segunda lengua o lengua extranjera (de aquí en adelante L2), y más aún cuando se trata de la producción de un texto académico.

Para el estudiantado de pedagogía en inglés en Chile es de vital importancia desarrollar habilidades avanzadas de escritura académica. Por una parte, se espera que puedan escribir textos como tesis de grado e investigaciones relativas a la enseñanza y al aprendizaje de la lengua inglesa, y por otra, el Ministerio de Educación de Chile estipula que el profesorado que egresa de los programas de pedagogía en inglés de las universidades chilenas deben acreditar un nivel $\mathrm{C} 1$ de acuerdo con el marco establecido por la comunidad Europea, el que se caracteriza por una alta precisión lingüística (Mineduc, 2014).

En este contexto, los resultados de las evaluaciones escritas del estudiantado de la carrera de Pedagogía en inglés de una universidad chilena dejan en evidencia, frecuentemente, la falta de precisión gramatical. Ello impide el logro de los objetivos en la escritura académica y resulta preocupante si se considera que el desarrollo de este tipo de textos es crucial en un contexto universitario.

Las razones de la baja calidad de los textos escritos en L2 del alumnado de pedagogía en inglés son diversas. Por una parte, se evidencia una falta de motivación para preparar tareas de redacción, debido a las dificultades propias de la escritura y a la falta de reflexión acerca de la importancia de desarrollar esta competencia gramatical. Por otra parte, el proceso de producción escrita requiere el manejo de una serie de competencias, entre ellas la competencia gramatical que involucra el uso correcto de tiempos verbales, conectores, preposiciones, entre otros, que muchas veces no han sido asimilados a cabalidad por los/las estudiantes antes de emprender procesos de escritura académica.

Algunas investigaciones señalan que personal docente de universidad en situaciones donde el idioma inglés no se encuentra en un contexto inmediato, como es el caso de Chile, consideran "los errores gramaticales como distractores y estigmatizadores, puesto que pueden interferir en la comprensión del mensaje" (Ferris y Robert, 2001, p.14). En concreto, se puede señalar que la precisión gramatical, es uno de los principales focos de atención del profesorado de inglés en Chile y, por esta razón, adquiere importancia en el presente estudio.

Otro aspecto problemático es el escaso impacto del feedback en los escritos del estudiantado, y que se observa en la reiteración de los mismos tipos de errores en sus textos académicos. Es por ello que este artículo aborda el uso del feedback indirecto metalingüístico, que, dada sus características, puede tener un impacto positivo en la corrección de errores.

Este artículo se inserta en el contexto del proyecto Fondecyt №11150273, denominado Uso de estrategias de feedback correctivo escrito focalizado en un entorno virtual colaborativo wiki: Impacto y plan de capacitación.

\section{MARCo TEÓRICo}

\section{Escritura académica}

La escritura académica se caracteriza por su precisión, objetividad y claridad y por el uso de un registro formal y un lenguaje especializado (Tapia, Burdiles y Arancibia, 2003; Neira y Ferreira 2011). El discurso académico tiene como uno de sus objetivos principales comunicar un descubrimiento científico. Otro de sus propósitos es convencer sobre la legitimidad e importancia científica de este nuevo conocimiento. Es decir, las funciones del texto académico son tanto informar cómo convencer. Para tal fin, el texto académico argumentativo ya no trata solo de exponer e informar sino de persuadir y convencer. 
Dentro de los principales tipos de texto académicos se pueden distinguir encontrar el resumen, el artículo científico y el ensayo. El ensayo, texto académico foco de este estudio, se caracteriza por la presentación de un tema desde una interpretación personal con rigor argumentativo. Es un género que permite cuestionar, ampliar o revisar puntos de vistas. Estructuralmente comprende los apartados: introducción, desarrollo y conclusión, a través de los cuáles se presentan la hipótesis, la tesis y la síntesis (Hyland, 1990).

El proceso de escritura de un ensayo, requiere el manejo de una serie de competencias, entre ellas la competencia gramatical que involucra el uso correcto de tiempos verbales, conectores, preposiciones, entre otros, que muchas veces no han sido asimilados a cabalidad por los/las estudiantes antes de emprender procesos de escritura académica. Al respecto, en el marco del aprendizaje de una L2, es justamente considerado un aspecto problemático el tema de la competencia gramatical, y más aún en un contexto donde el inglés es considerado una lengua extranjera. Si bien los errores gramaticales pueden no tener mayor incidencia en la transmisión de un mensaje, sí se considera una problemática en la escritura académica y más aún en un contexto universitario.

\section{Los errores en una $L 2$}

Todo estudiante, durante el proceso de adquisición de una L2, debe enfrentarse de manera frecuente a distintos tipos de obstáculos. Dentro de estos se pueden encontrar los errores lingüísticos que el estudiantado comete en sus producciones orales y escritas. Allwright y Bailey (2004) y Ellis (1995) definen un error como una desviación de las normas o formas correctas de una L2. A su vez, Brown (2000) señala que un error corresponde a una desviación evidente de la gramática y demuestra el nivel intelectual de quien aprende. Por otra parte, Blanco (2002) propone una definición más amplia y describe un error como "una transgresión, desviación o uso incorrecto de una norma, que en el caso que nos ocupa puede ser lingüística, pero también cultural, pragmática, y de una gran variedad de tipos más” (p. 20). Ferris (2013) plantea que "una forma común de priorizar un error es focalizarse en aquellos errores que el estudiantado comete frecuentemente" (p.88), por lo tanto, para efectos del presente estudio, un error es considerado como aquel que ocurre con mayor frecuencia en un texto escrito.

Un error se caracteriza por ser sistemático y, por lo tanto, es considerado significativo, pues demanda que el/la estudiante reciba algún tipo de feedback. Al respecto, la manera de corregir o entregar feedback a los errores frecuentes que comete el estudiantado en la escritura, es una de las tareas complejas para el personal docente, más aún, cuando se trata de la corrección de distintos tipos de errores, como sucede comúnmente en la producción escrita. Bustos (2006) plantea que una de las posibles causas de los errores reiterados que comete el alumnado es la ausencia de un feedback negativo o correctivo. Al no existir tal corrección, no detecta el error y lo repite.

\section{El feedback correctivo escrito y su rol en la adquisición de segundas lenguas}

Dentro de las estrategias de feedback, una de las más estudiadas y foco de interés para distintos teóricos es el feedback correctivo (FC) (Joy, 2005; Sheen, 2007; Tatawy, 2002 y otros), también denominado por la mayoría de los investigadores como 'evidencia negativa o feedback negativo'. Este tipo de feedback, que puede tener origen en el contexto natural o en el contexto del aula, es operacionalizado como el movimiento reactivo del y la docente, que invita al estudiante a atender a un error gramatical (Gass, Behney y Plonsky, 2013; Spada y Lightbown, 1999; Sheen, 2007).

El FC es generalmente propiciado por el cuerpo docente, este cuenta con la facultad y el rol de optar por distintas estrategias en el marco de la enseñanza y aprendizaje de una L2. A nivel de la enseñanza de una L2, el FC adquiere aún mayor importancia, esto debido a la dificultad que implica el desarrollo de competencias 
con respecto a otras en la adquisición del idioma inglés; como las gramaticales, por ejemplo. Por lo tanto, estas estrategias se convierten en una ayuda significativa que, por una parte, pueden potenciar los logros del estudiantado y, por otra, lo pueden orientar durante todo el proceso de aprendizaje.

El tratamiento de errores implica el uso de distintos tipos de feedback correctivo. Al respecto, algunos estudios empíricos (Bitchener, 2008; Bitchener y Knoch, 2008; 2009; Ferris, 2010; Ferris y Robert, 2001; Lee, 1997 y Sheen, 2007, 2010), si bien no son concluyentes, han demostrado que el feedback correctivo en el contexto de la producción escrita sí puede aportar en la adquisición de una L2. Dichos estudios dan cuenta del efecto de ciertos tipos de feedback correctivo en la adquisición de una L2, con una atención marcada en la forma. Estos tipos de feedback comprenden el feedback focalizado versus no focalizado, el feedback metalingüístico versus no metalingüístico y el feedback directo versus indirecto.

\section{Estrategias de feedback correctivo escrito focalizado y no focalizado}

El feedback focalizado significa que el personal docente corrige un error específico (Doughty y Williams, 2006; Han, 2002; Ellis, 2009). En cambio, el feedback no focalizado "implica entregar feedback a una amplia gama de errores identificados en los textos escritos de los/las estudiantes" (Sheen, Wright y Moldawa, 2009, p. 559). Esta última forma de entregar feedback ha sido el método más utilizado por los docentes en la corrección de textos escritos.

A la fecha, pocos estudios han comparado el uso del feedback focalizado con el no focalizado en la producción escrita. En 2007, Sheen investigó si existían diferencias significativas entre el feedback correctivo focalizado directo y el feedback correctivo no focalizado directo en el uso de formas gramaticales. El estudio arrojó que el feedback focalizado era más efectivo que el no focalizado. Sin embargo, Bitchener y Ferris (2011) también compararon los efectos del feedback focalizado y no focalizado en un estudio experimental con grupo control. Se concluyó que ambos grupos mejoraron la precisión lingüística de un texto escrito en el post-test.

Los estudios desarrollados a la fecha permiten concluir que no hay suficiente evidencia para afirmar que un tipo de feedback es más efectivo que otro y, por lo tanto, se requieren más investigaciones de este tipo. Pese a esta evidencia, Sheen (2010) afirma que "algunos estudios recientes destacan el valor del feedback correctivo escrito focalizado" (p. 11). Según la autora, el uso de feedback correctivo seguido de una explicación metalingüística sería el tipo de feedback más beneficioso en la corrección de aspectos gramaticales específicos en la producción escrita.

\section{Estrategias de feedback correctivo escrito metalingüístico versus no metalingüístico}

El feedback metalingüístico se define como aquel que entrega el/la docente con comentarios acerca de la lengua. Este tipo de feedback puede incluir solamente una explicación sobre la naturaleza del error o la explicación más la corrección del error (Nassaji y Fotos, 2011; Panova y Lyster 2002; Sheen, 2004).

Para efectos del presente estudio, por feedback metalingüístico debe entenderse el tipo de feedback en el cual el personal docente proporciona una explicación gramatical del error. En cambio, el feedback no metalingüístico implica entregar la respuesta correcta al estudiante sin proporcionar ninguna explicación adicional. Al respecto, Ellis (2006) y Sheen (2010) afirman que el conocimiento explícito proporcionado a través del feedback metalingüístico puede apoyar a los/las estudiantes en el desarrollo de la L2. Así, la entrega de este tipo de feedback los ayudaría, por una parte, a reflexionar sobre sus errores y, por otra, a comprender la naturaleza de los mismos.

Algunos autores han afirmado que el feedback correctivo metalingüístico es superior a uno sin la explicación metalingüística. En este contexto, Bitchener (2008), Bitchener y Knoch (2009) y Sheen (2010) 
han concluido que el uso del feedback escrito, apoyado por una explicación metalingüística, puede tener un efecto beneficioso en la adquisición de determinadas estructuras lingüísticas. En concreto, el componente metalingüístico implica la capacidad del estudiantado de reflexionar sobre el lenguaje e ir más allá del uso de la lengua.

De acuerdo a Gass et ál. (2013) la habilidad para reflexionar sobre el lenguaje se asocia con una mayor habilidad para aprender un idioma. En otras palabras, la atención se dirige al lenguaje mismo y conlleva una reflexión más profunda y más consciente sobre lo que se está aprendiendo. a esta conciencia metalingüística.

\section{Estrategias de feedback correctivo escrito directo e indirecto}

La diferencia entre el feedback directo y el indirecto radica en que, en el primero, el cuerpo docente identifica el error y entrega al estudiantado la forma correcta, mientras que en el segundo, cada docente indica a su estudiante que ha cometido un error (Ellis, 2006), pero sin entregar la forma correcta.

En relación con estos dos tipos de feedback, algunos estudios revelan que el estudiantado y el profesorado muestra mayor preferencia por el feedback directo (Ferris y Roberts, 2001; Komura, 1999; Rennie, 2000). Sin embargo, los estudios plantean que el feedback indirecto puede tener, con el transcurso del tiempo, igual o mejores efectos en la precisión lingüística, que el feedback directo (Ferris y Helt, 2000; Frantzen, 1995; Jhwory, 2010). Del mismo modo, algunos autores señalan que el feedback indirecto cambiaría la percepción del estudiantado sobre la responsabilidad en su propio aprendizaje (Chandler, 2003; Srichanyachon, 2012). En este contexto, el uso del feedback indirecto promovería el pensamiento crítico en el/la estudiante y contribuiría a que su rol sea mucho más activo.

Ferris y Helt (2000) ejecutaron un estudio en el que demostraron que la corrección directa incentivaba al estudiantado a revisar y efectuar modificaciones en sus escritos con mayor frecuencia que el feedback indirecto. Sin embargo, después de la aplicación de un post-test diferido se demostró que gracias al feedback indirecto el estudiantado disminuyó la cantidad de errores en sus escritos. Asimismo, estudios recientes llevados a cabo por Bitchener y Knoch (2010) afirman que tanto el feedback indirecto como el directo son efectivos a corto plazo. A su vez, Van Beuningen, De Jong y Kuiken (2012) señalan que, en el largo plazo, el feedback directo sería más efectivo que el feedback indirecto. Frantzen (1995) y Robb, Ross y Shortreed (1986), por su parte, afirman que el feedback directo e indirecto serían igualmente efectivos.

De las investigaciones llevadas a cabo en el contexto del feedback correctivo, hay que considerar que algunas de ellas no han contado con un grupo control, por ende, sus resultados no serían del todo confiables. Esta ausencia del grupo control se debe a ser considerado poco ético para algunos teóricos dejar a un grupo sin corregir, dado que "el estudiantado tiene el derecho de ser corregido y probablemente espera ser corregido" (Sheen, 2007, p. 92). Lo anterior es una de las razones fundamentales que impiden que se lleve a cabo una investigación experimental real. No obstante, existen investigaciones que sí han logrado incorporar grupos de control.

En relación con lo anterior, estudios llevados a cabo por Ferris y Robert (2001), Robb et ál. (1986) y Semke (1984) sí contaron con un grupo control el cual no recibió feedback. Dentro de estos estudios, tres trabajos demostraron que el grupo experimental obtuvo mejores resultados que el grupo control, aunque las diferencias no fueron significativas. Así, Lee (1997) encontró efectos significativos en un estudio con grupos de estudiantes de inglés como lengua extranjera, cuyos errores fueron subrayados, a diferencia del grupo que no recibió feedback correctivo. Al respecto, Sheen (2007) señala que algunos estudios que han contado con grupo control, por razones éticas, han recibido algún tipo de feedback, como por ejemplo, comentarios acerca del contenido y la organización del texto.

Otro aspecto que genera discusión es la medición del efecto del feedback correctivo. En algunos estudios, el objetivo ha sido comprobar si el estudiantado ha realizado las correcciones en su primer borrador (Fathman y Whalley, 1990; Ferris y Roberts, 2001; Oubourmerrad, 2013; Reitbauer, Campbell, Mercer, Schumm y 
Vaupetitsch, 2013). Sin embargo, pocos estudios han medido el progreso en la producción escrita en términos de precisión lingüística (Ashwell, 2000; Robb et ál. 1986), así como tampoco, el efecto del feedback escrito de manera longitudinal, es decir, por un periodo largo de tiempo (Van Beuningan et ál. 2012; Chandler, 2003; Kepner, 1991).

Las investigaciones que han observado el efecto del feedback correctivo por un periodo de tiempo breve señalan que este puede ayudar al estudiantado a mejorar su precisión lingüística en un texto escrito. Sin embargo, tales estudios no han estado exentos de crítica, puesto que no demuestran un progreso de manera longitudinal (Truscott, 1996; Truscott y Hsu, 2008). Ferris (2010) señala que los estudios longitudinales no son posibles si no existe primero una intervención breve, por lo tanto, "si bien estos estudios no responden a todas las inquietudes, no pueden ser ignorados" (p. 13).

Para entregar feedback correctivo a un texto escrito existen diferentes maneras de proceder. Ferris (2010) presenta el siguiente modelo para la revisión de la producción escrita:
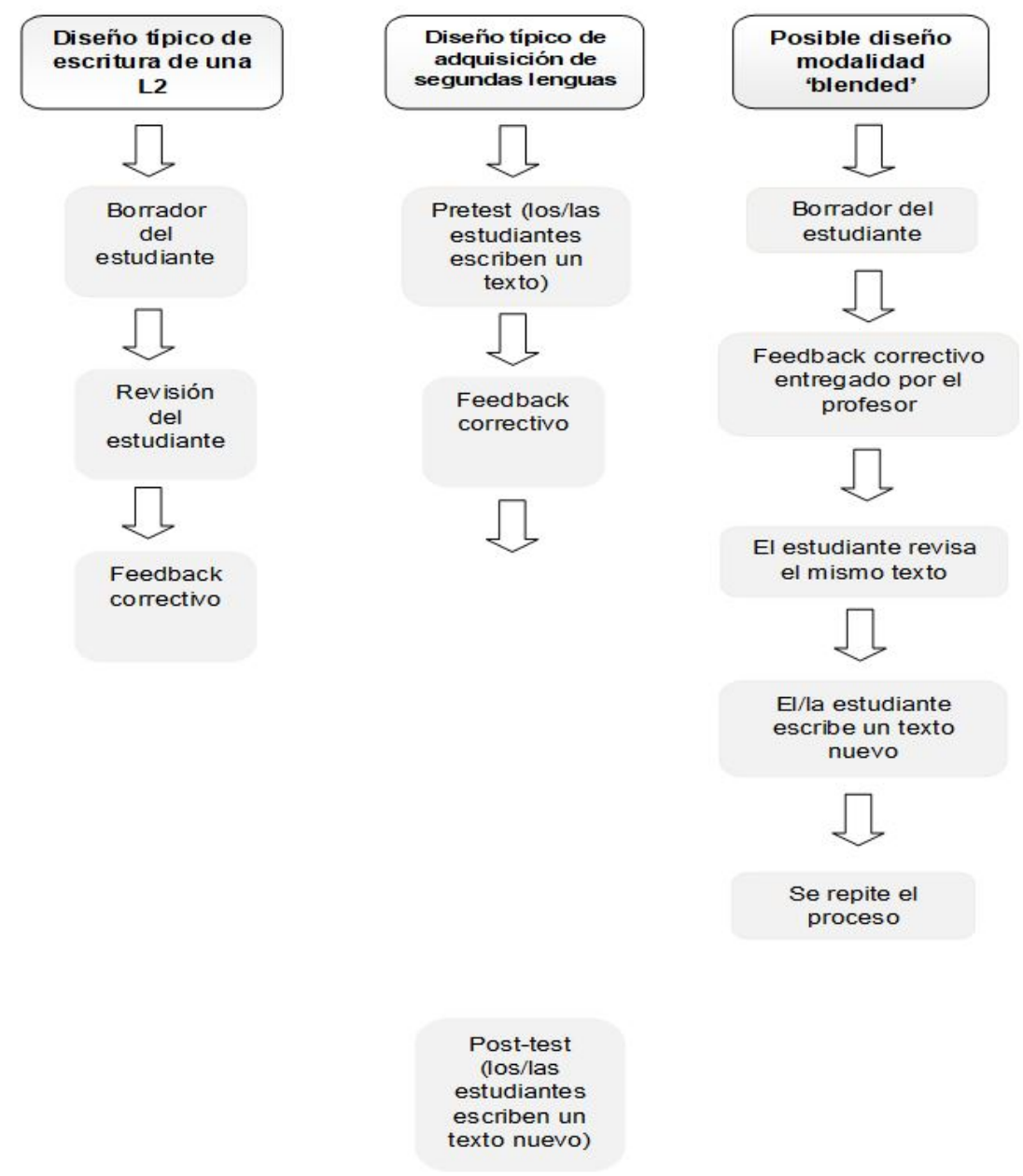

FIGURA 1

Modelo de revisión de producción escrita

Fuente: Ferris (2010, traducción de los autores)

Como muestra la Figura 1, en el primer modelo se escribe un solo texto. El y la estudiante lo revisa y, posteriormente, recibe feedback. En el segundo modelo, se incorpora un pretest escrito, se recibe feedback y en el post-test se escribe un texto nuevo. En este modelo se sugiere también un post-test diferido, como se ha observado en algunas investigaciones de Sheen (2007), Bitchener (2008) y Bitchener y Knoch (2009). En relación con el modelo mixto, el y la estudiante debe revisar su escrito después del feedback entregado por el 
o la docente y, posteriormente, escribir un nuevo texto con el fin de repetir el proceso nuevamente. En este último modelo, el estudiantado recibe feedback en repetidas ocasiones.

En el presente estudio, se ha optado por el modelo mixto. Ello dado que se considera relevante que el estudiantado revise el texto después del feedback entregado por el personal docente, proceden a corregir los errores y luego escriben otro tema sobre un tópico distinto. En efecto, la revisión se considera parte del proceso de escritura y es significativa en cuanto permite internalizar el feedback elaborado por el cuerpo docente.

\section{Metodología}

\section{Pregunta de investigación}

¿Cuál es el impacto del feedback indirecto metalingüístico en inglés en la reducción del número de errores gramaticales en textos argumentativos breves escritos por estudiantes de tercer año de pedagogía en inglés de una universidad chilena?

\section{Hipótesis}

Para dar respuesta a la pregunta de investigación se planteó la siguiente hipótesis de investigación: La provisión de feedback indirecto metalingüístico tiene un impacto positivo en la reducción del número de errores gramaticales en textos argumentativos breves escritos por los/las estudiantes de tercer año de pedagogía en inglés de una universidad chilena.

\section{Diseño de la investigación}

El diseño de la investigación correspondió a un pre-experimento con pre y post test, donde se midió el desempeño del estudiantado al escribir ensayos en inglés antes y después del tratamiento.

\section{Muestra}

Se utilizó una muestra no probabilística intencionada de 20 estudiantes, 10 varones y 10 mujeres, pertenecientes a la carrera de Pedagogía Media en Inglés de una universidad chilena.

Las edades de los participantes fluctuaban entre 20 y 22 años.

El nivel de competencia lingüística de los participantes corresponde a un nivel intermedio alto (B2), según el Marco Europeo de Referencias para las Lenguas.

\section{Instrumentos}

Para efectos del estudio se utilizaron los siguientes instrumentos:

Cuestionario sociodemográfico: Este instrumento fue utilizado para recoger antecedentes diversos de la muestra, tales como: edad, estudios secundarios, horas de inglés a la semana en el plan de estudio de enseñanza media, etc.

Tabla de categorías de errores. Este instrumento se utilizó para la elicitación del error. Se adjunta Tabla 1 
TABLA 1

Tabla de categorías de errores

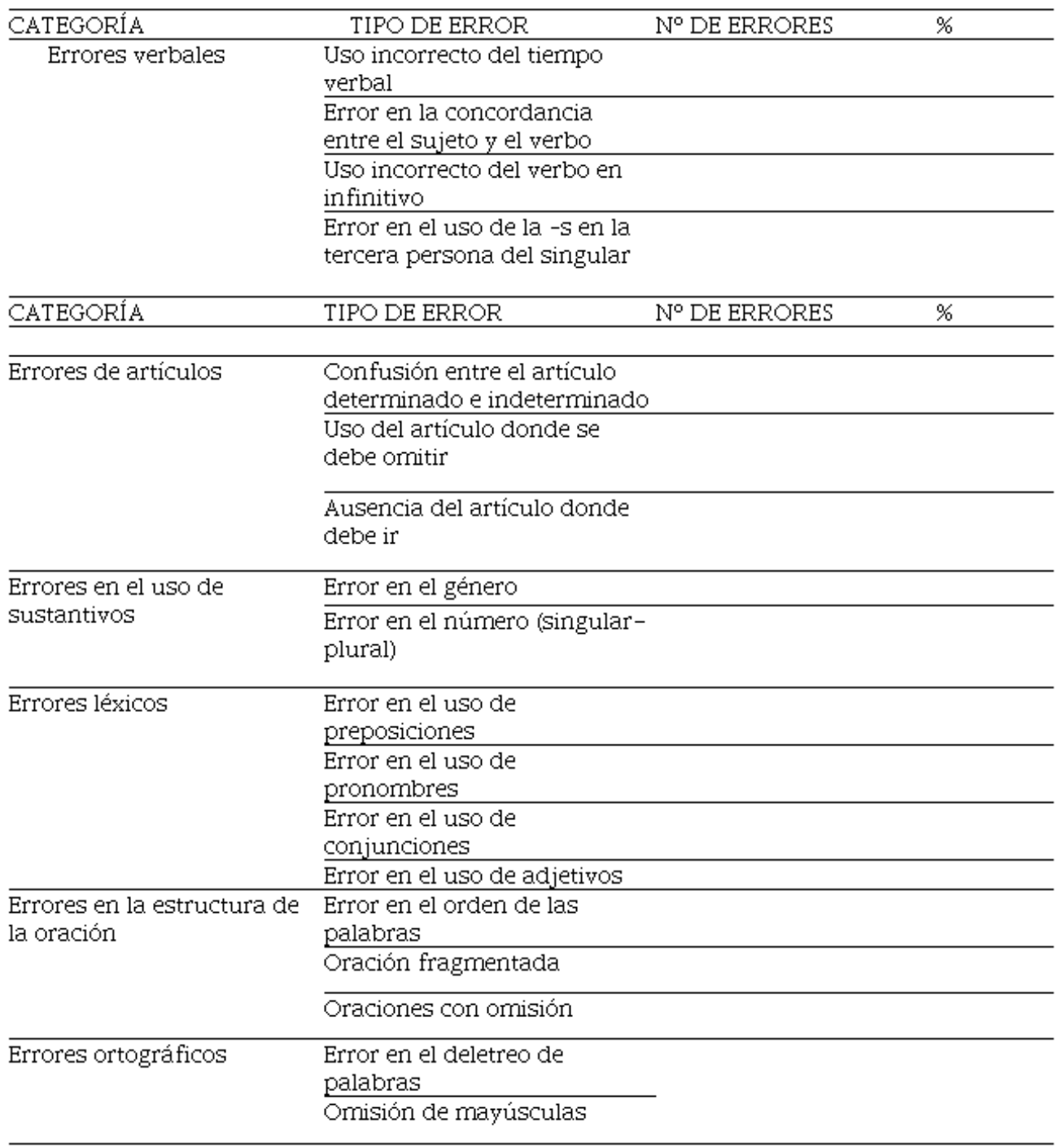

Fuente: Adaptada de Ferris (1999) y Bitchener, Young y Cameron (2005).

1. Pretest-postest: El instrumento utilizado correspondió a una parte de la sección escrita del instrumento de medición TOEFL (Test of English as a Foreing Language).

2. Tareas de escritura: Las tareas consistieron en el desarrollo de cuatro ensayos argumentativos breves (300 palabras).

\section{Validez de los instrumentos}

Cada instrumento fue sometido al juicio de seis docentes de inglés de una universidad chilena. Cada uno de ellos verificó que los instrumentos apuntaran a los objetivos de investigación planteados. Para tales efectos, se diseñó una pauta en el que cada experto debía especificar el grado de acuerdo respecto a las instrucciones y los ítems de cada instrumento. 


\section{Procedimiento}

La intervención se estructuró en tres etapas:

\section{Etapa I}

En la primera fase de esta investigación, se seleccionó la muestra intencionada compuesta por 20 sujetos y se aplicó un pretest. Todos ellos eran estudiantes de tercer año de la carrera de pedagogía en inglés de una universidad chilena.

\section{Etapa II}

En la segunda fase se aplicó el tratamiento por un periodo de tres meses. Este consistió en la creación individual de cuatro tareas de escritura de textos argumentativos breves.

En el afán de comprender el procedimiento que se debe seguir en el proceso de escritura, la Figura 2 muestra el proceso de escritura de cada texto argumentativo.

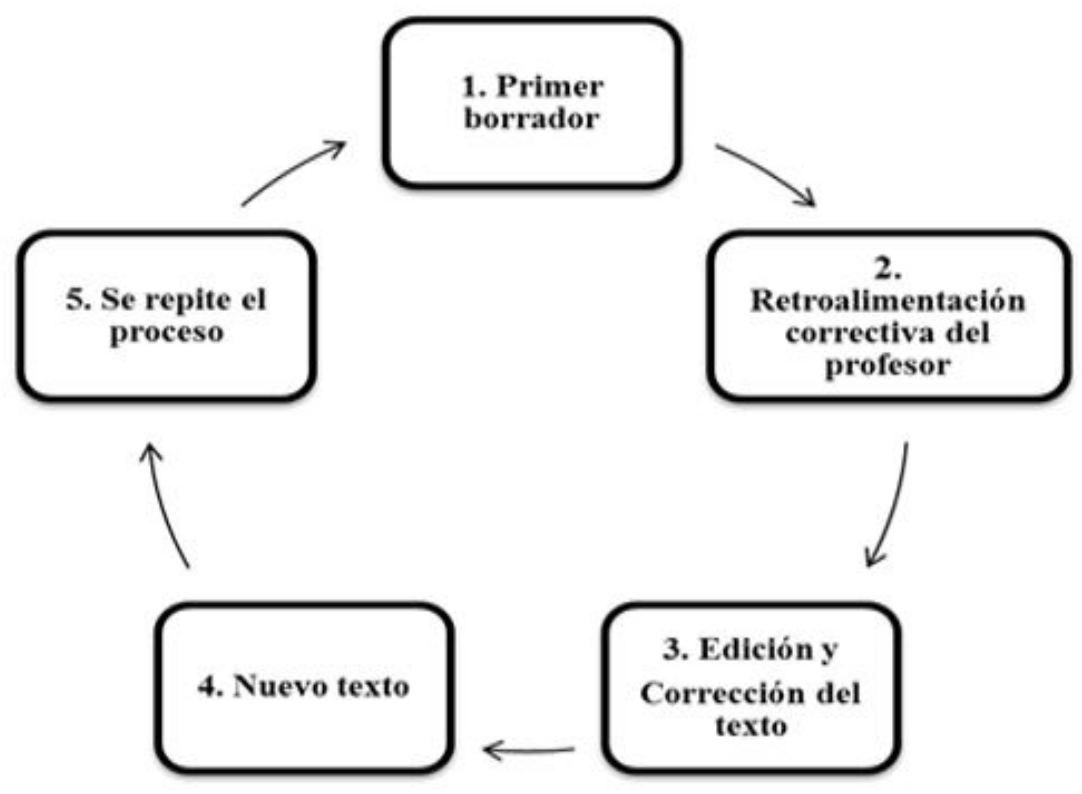

FIGURA 2

Proceso de escritura de un texto

Fuente: Elaboración propia

La Figura 2 describe las etapas de la escritura en proceso de un texto argumentativo. Primero, el estudiantado escribe su primer borrador. Luego, reciben feedback correctivo por parte del y la docente. Posteriormente, corrigen sus errores y escriben un nuevo texto. Finalmente, se repite el proceso.

\section{Etapa III.}

Se aplicó un post-test con el fin de determinar si hubo un avance en términos de competencia gramatical en los textos de los participantes después de la intervención. 


\section{ANÁLISIS DE LOS DATOS}

Para verificar si estadísticamente disminuyó la cantidad de errores cometidos por las y los alumnos, al comparar los resultados del primer y cuarto ensayo, se realizaron dos procedimientos:

I. En primera instancia se verificó si disminuyó la cantidad de errores cometidos por las y los alumnos de manera general en los ensayos.

II. Finalmente se verificó si disminuyó la cantidad de errores cometidos por las y los alumnos haciendo diferencia entre los tipos de error.

Para efectuar el análisis de los datos se utilizó el programa SPSS y se aplicó la prueba estadística de Wilcoxon. Esta se utiliza para comparar la media de dos muestras relacionadas y determinar si existen diferencias o no entre ellas. Debido a que la muestra de estudio es de tipo intencional, se optó por aplicar una prueba de carácter no paramétrica.

\section{Resultados}

\section{Por errores en general:}

$\mathrm{Al}$ analizar la cantidad de errores cometidos de manera general por parte de las y los alumnos, en la Figura 3 se aprecia claramente una disminución de estos en la redacción del cuarto ensayo.

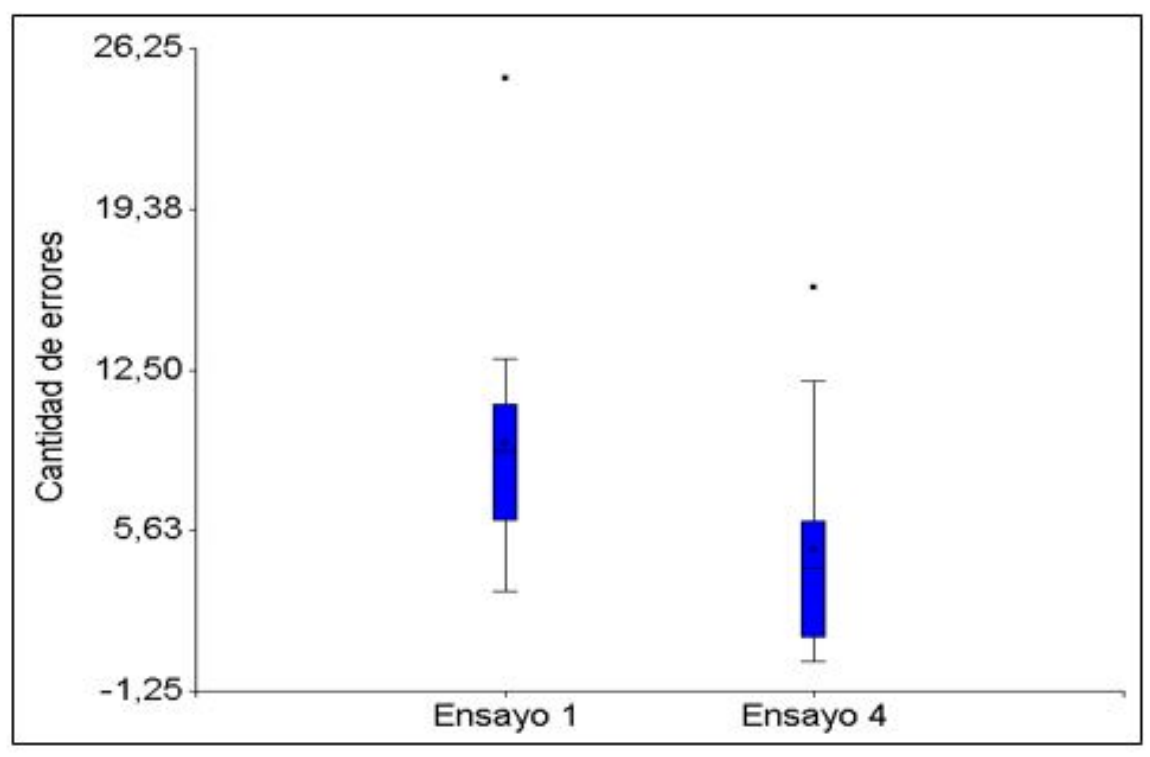

FIGURA 3

Distribución de la cantidad de errores cometidos en cada ensayo

Fuente: Elaboración propia

Posteriormente para verificar que esta diferencia es estadísticamente significativa se ejecutó la prueba no paramétrica de rangos con signos de Wilcoxon (valor-p=0,004), tal como lo muestra la Tabla 2.

Los resultados demuestran que el feedback recurrente sí influye en las y los alumnos a la hora de escribir sus ensayos, dado que los ayudaría a cometer menos errores. 
TABLA 2

Estadísticos de contraste

\begin{tabular}{ll}
\hline & Ensayo 4 - Ensayo 1 \\
\hline$Z$ & $-2,782 \mathrm{~b}$ \\
Sig. asintót. (bilateral) &, 005 \\
\hline
\end{tabular}

Fuente: Elaboración propia

\section{Por categorías de errores:}

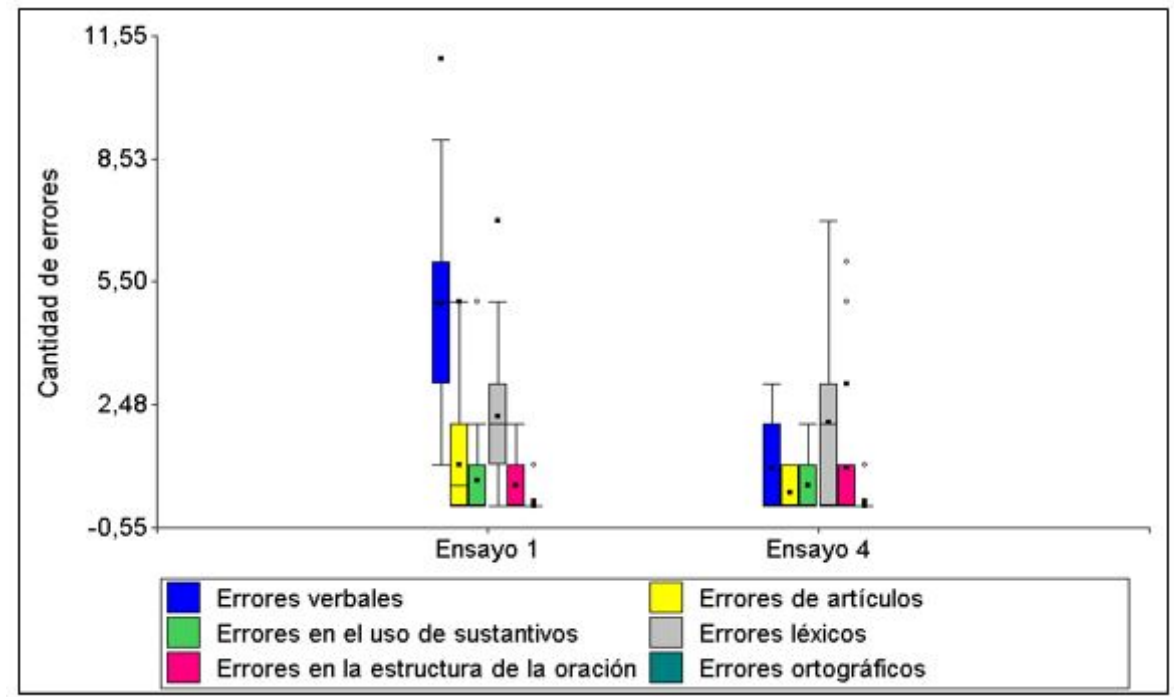

FIGURA 4

Distribución de la cantidad de errores cometidos por tipo de error Fuente: Elaboración propia

$\mathrm{Al}$ analizar la cantidad de errores cometidos por tipo de categorías de errores en la Figura 4 se aprecia que los errores verbales (uso incorrecto del tiempo verbal, error de concordancia sujeto-verbo, uso incorrecto del verbo en infinitivo y error en la tercera persona singular), y de artículos (confusión entre el artículo determinado e indeterminado, uso del artículo donde se debe omitir y ausencia del artículo donde debe ir), disminuyeron en la evaluación final. Además, se observa un comportamiento más bien constante en casi todas las otras categorías de errores.

Por otra parte, en relación a los errores léxicos, se observa que estos aumentaron en la redacción del cuarto ensayo.

Para comprobar si las diferencias entre un test y otro resultaron significativas se utilizó nuevamente la prueba no paramétrica de rangos con signos de Wilcoxon.

En la tabla 3 se encuentran los resultados de la prueba de rangos con signo de Wilcoxon, la cual contrasta la influencia del feedback en la escritura de los ensayos. Se observa que existe diferencia significativa en el caso de la categoría de errores verbales y de artículos. Es decir, en esos casos el feedback indirecto sí influye en las y los alumnos a la hora de escribir sus ensayos, por lo tanto, los ayuda a cometer menos errores. Por otra parte, en las demás categorías de errores no se encuentra evidencia del efecto del feedback. 
TABLA 3

Estadísticos de contraste

\begin{tabular}{lll}
\hline Tipo de error & $\mathrm{Z}$ & Sig. asintót. (bilateral) \\
\hline Errores verbales & 3,756 &, 000 \\
Errores de artículos & 2,169 &, 030 \\
Errores en el uso de sustantivos & 0,424 &, 672 \\
Errores léxicos & 0,454 &, 650 \\
Errores en la estructura de la & 0,992 &, 321 \\
oración & 0 & 1,000 \\
Errores ortográficos & 0 &
\end{tabular}

Fuente: Elaboración propia

$\mathrm{Al}$ ser estos resultados estadísticamente significativos podemos concluir que la hipótesis planteada en este trabajo es válida.

\section{Discusión}

Al comparar los resultados entre el pretest y posttest se puede señalar que el feedback indirecto metalingüístico sí tuvo impacto positivo en los resultados, puesto que se observó una disminución de errores después del tratamiento. Desde una perspectiva teórica, los resultados pueden tener diversas explicaciones, dado que más de un factor pudo haber incidido en las diferencias de errores entre el pretest y el posttest. En este contexto, se puede inferir que un factor que puede haber incidido en el avance del grupo en el posttest es la sistematicidad y la frecuencia con que se aplicó el feedback indirecto metalingüístico. Como lo afirma Ferris (1999, 2002, 2003), el hecho de que el feedback sea entregado de manera frecuente puede influir positivamente en el uso adecuado de las formas lingüísticas. De esta manera que el estudiantado tenga mayores oportunidades para prestar atención a las estructuras lingüísticas, aumenta las probabilidades de que utilicen las estructuras de forma adecuada al escribir un nuevo texto.

Se destaca también del tratamiento llevado a cabo, las fases de revisión y de reescritura de un mismo texto y la posibilidad de otorgar al estudiante la producción de nuevo texto, las que otorgan al estudiante mayores oportunidades de análisis, de reflexión y de corrección de errores. Esta práctica de escribir un nuevo texto, no siempre es implementada, dado el tiempo prolongado que ocupa la actividad de escritura.

Otro factor importante que puede haber incidido en la disminución de errores es la provisión de un feedback con claves metalingüísticas. Este tipo de elemento que acompaña al feedback puede propiciar la reflexión y la búsqueda de respuestas frente a dudas sobre la lengua que se estudia. Por lo tanto, se infiere que este tipo de feedback puede tener particularmente un impacto positivo en el estudiantado que posee un estilo de aprendizaje más autónomo y al mismo tiempo más reflexivo o, dicho de otro modo, que procesan mejor la información a través del análisis. Así, la reflexión metalingüística puede contribuir a un aprendizaje más eficaz de la lengua. Esto es corroborado por Gass et ál. (2013), quien declara que las estrategias que apuntan a la reflexión sobre el lenguaje, se asocian con una mayor habilidad para aprender un idioma.

La no provisión de claves metalingüísticas puede ser una de las causas importantes que explican el por qué el estudiantado sigue cometiendo los mismos tipos de errores en sus textos. Al respecto, no es común que el estudiantado reciba explicaciones metalingüísticas sobre sus errores y, en el caso de que se hagan, estas no son frecuentes ni sistemáticas. Por lo tanto, se puede deducir que la utilización de claves metalingüísticas en un estudiante que adquiere el idioma como parte de su formación de docente de inglés es aún más relevante, puesto que el futuro docente no solamente debe fortalecer el conocimiento pragmático de la lengua, sino también el conocimiento declarativo. En otras palabras, la competencia en el uso de la gramática de un futuro docente de inglés debe ser superior a la de un estudiante que adquiere una L2 con fines comunicativos. 


\section{Conclusiones}

A la luz de los resultados, y respondiendo al objetivo de este estudio, el cual buscaba determinar el impacto del feedback indirecto metalingüístico en inglés en la reducción del número de errores gramaticales en textos argumentativos breves, se puede concluir primero que el feedback es una estrategia relevante en la corrección de errores y especialmente en contextos donde el inglés es una considerada una lengua extranjera.

Segundo, el tipo de feedback correctivo puede influir en la corrección de errores. En el caso del presente estudio el feedback indirecto metalingüístico posee características que pueden aportar al tratamiento de algunos tipos de errores. Se concluye que un feedback acompañado de información metalingüística es una oportunidad para que el estudiantado adquiera un conocimiento de la lengua dentro de una situación comunicativa. Por consiguiente, el conocimiento de reglas gramaticales no tiene que ser ignorado de la enseñanza de lenguas ni tampoco puede ser desvinculado de un proceso comunicativo, más aún si se persigue un fin pedagógico. La gramática es más que solo reglas y, por ende, es más útil y significativa para el alumnado la enseñanza en situaciones comunicativas que de manera aislada.

Tercero, se puede concluir también que algunos elementos que pueden contribuir a mejorar la calidad de la escritura académica de textos argumentativos breves son la sistematicidad y frecuencia del feedback correctivo. En otras palabras, el impacto del feedback que entrega el cuerpo docente depende significativamente del grado de frecuencia y sistematicidad con que se utilice. Por lo tanto, es un aspecto a considerar en la enseñanza y aprendizaje del inglés, principalmente en la corrección de errores específicos de la lengua. Finalmente, se considera relevante también otorgar al estudiantado la posibilidad de re-escribir su texto y posteriormente escribir un nuevo texto.

\section{REFERENCIAS}

Allwright, D. y Bailey, K. (2004). Focus on the language classroom. Nueva York: Cambridge University Press.

Arias, O. y García, J. (2006). El papel de la revisión en los modelos de escritura. Aula Abierta, 88, 37-52.

Ashwell, T. (2000). Patterns of teacher response to student writing in a multiple-draft composition classroom: Is content feedback followed by form feedback the best method? Journal of Second Language Writing, 9(3), 227-257.

Bitchener, J. y Ferris, D. (2011). Written Corrective Feedback in Second Language Acquisition and Writing. Nueva York: Routledge, Taylor y Francis Group.

Bitchener, J. (2008). Evidence in support of written corrective feedback. Journal of Second Language Writing, 17(2), $102-118$.

Bitchener, J., y Knoch, U. (2008). The value of written corrective feedback for migrant and international students. Language Teaching Research, 12(3), 409-431.

Bitchener, J., y Knoch, U. (2009). The relative effectiveness of different types of direct written corrective feedback. System, 37(2), 322-329.

Bitchener, J., y Knoch, U. (2010). The contribution of written corrective feedback to language development: a ten month investigation. Applied Linguistics, 31(2), 193-214.

Bitchener, J., Young, S., y Cameron, D. (2005). The effect of different types of corrective feedback on ESL student writing.Journal of Second Language Writing, 14(3), 191-205.

Blanco, A. (2002): El error en el proceso de aprendizaje. Cuadernos Cervantes de la lengua español, 8(38), 12-22.

Brown, H. (2000). Principles of Language Learning and Teaching. New York: Addison Wesley Longman Inc.

Bustos, J. M. (2006). Principios metodológicos para el estudio de la ¿fosilización? en el aprendizaje del español como lengua extranjera: el buen aprendiz. En J. M. Bustos y J. Sánchez Iglesias (Coords.), La estabilización en el aprendizajeladquisición del español como L2: el buen aprendiz (pp. 59-102). Salamanca: Hispano Lusa de Ediciones. 
Mabel Ortiz Navarrete, et al. Uso del feedback correctivo indirecto metalingüístico y SU EFECTo E...

Chandler, J. (2003). The efficacy of various kinds of error feedback for improvement in the accuracy and fluency of L2 student writing. Journal of second language writing, 12(3), 267-296.

Doughty, C. y Williams, J. (2006). Focus on form in classroom second language acquisition. Cambridge: Cambridge University Press.

Ellis, R. (1995). Interpretation tasks for grammar teaching. TESOL Quarterly, 29, 87-105.

Ellis, R. (2006). Researching the effects of form-focused instruction on L2 acquisition. AILA Review, 19(1), 18-41.

Ellis, R. (2009). A typology of written corrective feedback types. ELT journal, 63(2), 97-107.

Fathman, A. y Whalley, E. (1990). Teacher response to student writing: Focus on form versus content. En B. Kroll (ed.) Second language writing (Cambridge Applied Linguistics): Research insights for the classroom. pp. 178-190. Cambridge: Cambridge University Press.

Ferris, D. (1999). The case for grammar correction in L2 writing classes: A response to Truscott. Journal of Second Language Writing, 8(1), 1-11.

Ferris, D. (2002). Treatment of error in second language student writing. Estados Unidos: University of Michigan Press. Ferris, D. (2003). Response to student writing: Implications for second language students. Mahwah, NJ: Erlbaum.

Ferris, D. (2010). Second language writing research and written corrective feedback in SLA. Studies in Second Language Acquisition, 32(2), 181-201.

Ferris, D. (2013). What L2 writing means to me: Texts, writers, contexts. Journal of Second Language Writing, 22(4), 151-179.

Ferris, D. y Helt, M. (2000). Was Truscott right? New evidence on the effects of error correction in L2 writing classes. En A. Collins (Presidencia). American Association of Applied Linguistics Conference. Conferencia llevada a cabo en Vancouver, BC.

Ferris, D. y Roberts, B. (2001). Error feedback in L2 writing classes: How explicit does it need to be? Journal of second language writing, 10(3), 161-184.

Frantzen, D. (1995). The effects of grammar supplementation on written accuracy in an intermediate Spanish content course. The Modern Language Journal, 79(3), 329-344.

Gass, S., Behney, J. y Plonsky, L. (2013). Second language acquisition: An introductory course. New York: Routledge.

Han, Z. (2002). A study of the impact of recasts on tense consistency in L2 output. Tesol Quarterly, 36(4), 543-572.

Hyland. K. (1990). A genre description of the argumentative essay. ELC Journal: A Journal of Language Teaching and Research in Southeast Asia, 21(1), 66-78

Jhwory, K. (2010). Does the provision of an intensive and highly focused indirect corrective feedback lead to accuracy? (Tesis de Maestría). University of North Texas, Texas.

Joy, R. (2005). The effect of feedback and instruction on writing quality: Legal writing and advanced L2 learners (Tesis de Doctorado). Washington University, Washington D.C.

Mineduc. (2014). Estándares orientadores para carreras de Pedagogia Media en Inglés. Recuperado de: https://bibliot ecadigital.mineduc.cl/handle/20.500.12365/2237

Kepner, C. (1991). An Experiment in the Relationship of Types of Written Feedback to the Development of Second\# Language Writing Skills. The Modern Language Journal, 75(3), 305-313

Kessler, G. (2009). Student-initiated attention to form in wiki-based collaborative writing. Language. Learning $y$ Technology, 13(1), 79-95.

Komura, K. (1999). Student response to error correction in ESL classrooms (Tesis de Maestría). California State University. Sacramento.

Lee, I. (1997). ESL learners' performance in error correction in writing: Some implications for teaching. System, 25(4), 465-477.

Myles, J. (2002). Second language writing and research: The writing process and error analysis in student texts. TESLEJ, 6(2), 1-20. 
Nassaji, H. y Fotos S. (2011). Teaching grammar in second language classrooms: Integrating form-focused instruction in communicative context. Londres: Routledge.

Neira, A. y Ferreira, A. (2011). Escritura académica: un modelo metodológico efectivo basado en tareas y enfoque cooperativo. Literatura y lingüistica, 24, 143-159.

Oubourmerrad, M. (2013). Communicative dimensions of corrective feedback in the EFL classroom. Alemania: Lambert Academic Publishing.

Panova, I. y Lyster, R. (2002). Patterns of corrective feedback and uptake in an adult ESL classroom. TESOL Quarterly, $36(4), 573-595$.

Reitbauer, M., Campbell, N., Mercer, S., Schumm, J., Vaupetitsch, R. (2013). Feedback matters. Current feedback practices in the EFL classroom. Nueva York: Peter Lang Edition.

Rennie, C. (2000). Error feedback in ESL writing classes: What do students really want. (Tesis de Maestría). University of Sacramento, California

Robb, T., Ross, S. y Shortreed, I. (1986). Salience of feedback on error and its effect on EFL writing quality.TESOL Quarterly, 20(1), 83-95.

Semke, H. (1984). Effects of the red pen. Foreign Language Annals, 17(3), 195-202.

Sheen, Y. (2004). Corrective feedback and learner uptake in communicative classrooms across instructional settings. Language Teaching Research, 8(3), 263-300.

Sheen, Y. (2007). The effect of focused written corrective feedback and language aptitude on ESL learners' acquisition of articles. TESOL Quarterly, 41(2), 255-283.

Sheen, Y. (2010). Differential effects of oral and written corrective feedback in the ESL classroom. Studies in Second Language Acquisition, 32(2), 201-234.

Sheen, Y. Wright, D. y Moldawa, A. (2009). Differential effects of focused and unfocused written correction on the accurate use of grammatical forms by adult ESL learners. System 37, 556-569.

Spada, N. y Lightbown, P. (1999). Instruction, first language influence, and developmental readiness in second language acquisition. The Modern Language Journal, 83(1), 1-22.

Srichanyachon, N. (2012). Teacher written feedback for L2 learners' writing development. Journal of Social Sciences, Humanities and Arts, 12(1), 7-17.

Tatawy, M. (2002). Corrective feedback in second language acquisition, Columbia University. Working Papers in TESOL y Applied Linguistics, 2(2), 1-19.

Tapia, M., Burdiles, G. y Arancibia, B. (2003). Aplicación de una pauta diseñada para evaluar informes académicos universitarios. Signos, 36(54): 249-257.

Truscott, J. (1996). The case against grammar correction in L2 writing classes. Language learning, 46(2), 327-369.

Truscott, J. y Hsu, A. (2008). Error correction, revision, and learning. Journal of Second Language Writing, 17, 292-305.

Van Beuningen, C. G., De Jong, N. H., y Kuiken, F. (2012). Evidence on the effectiveness of comprehensive error correction in second language writing. Language Learning, 62(1), 1- 41.

Warschauer, M. (2010). Invited commentary: New tools for teaching writing. Language Learning and Technology, $14(1), 3-8$.

CC BY-NC-ND 